\title{
Gravitational scattering by giant planets
}

\author{
T. Laakso $^{1}$, J. Rantala ${ }^{2}$, and M. Kaasalainen ${ }^{1}$ \\ 1 Department of Mathematics and Statistics, Rolf Nevanlinna Institute, PO Box 68, 00014 University of Helsinki, Finland \\ e-mail: teemu.laakso@helsinki.fi \\ 2 Observatory, PO Box 14, 00014 University of Helsinki, Finland
}

Received 1 March 2006 / Accepted 25 May 2006

ABSTRACT

\begin{abstract}
We seek to characterize giant-planet systems by their gravitational scattering properties. We do this to a given system by integrating it numerically along with a large number of hypothetical small bodies that are initially in eccentric habitable zone (HZ)-crossing orbits. Our analysis produces a single number, the escape rate, which represents the rate at which the small-body flux is perturbed away by the giant planets into orbits that no longer pose a threat to terrestrial planets inside the HZ. Obtaining the escape rate this way is similar to computing the largest Liapunov exponent as the exponential rate of divergence of two nearby orbits. For a terrestrial planet inside the HZ, the escape rate value quantifies the "protective" effect that the studied giant-planet system offers. Therefore, escape rates could provide information on whether certain giant-planet configurations produce a more desirable environment for life than the others. We present some computed escape rates on selected planetary systems, focusing on effects of varying the masses and semi-major axes of the giant planets. In the case of our Solar System we find rather surprisingly that Jupiter, in its current orbit, may provide a minimal amount of protection to the Earth.
\end{abstract}

Key words. stars: planetary systems - solar system: general - celestial mechanics - methods: numerical - astrobiology

\section{Introduction}

The fascinating dynamical variety of extrasolar planetary systems has been the motivation behind many recent numerical simulations. We have seen detailed studies concerning the orbital stability of observed multi-planet systems (e.g., Barnes \& Quinn 2004) and also stability analyses where fictitious terrestrial planets are integrated along with the observed giant-planet system (e.g., Jones et al. 2005; Menou \& Tabachnik 2003; Asghari et al. 2004). In a few cases (GJ 777A, 47 UMa, HD 4208, HD 72659 etc.) it is found that terrestrial planets could indeed survive inside the habitable zone (HZ) of the system in a million year time scale.

For those candidate systems that could harbor Earth-like planets, we can take our dynamical speculation one step further and ask: How intense would the small-body flux be in the habitable zone of the planetary system in question? After all, small-body impacts to the Earth have had a major influence on the evolution of terrestrial life. Although it is arguable whether a smaller or larger small-body flux would have been more beneficial for our evolution, it would still be a step forward if we could present something quantitative about the small-body fluxes in the extrasolar systems.

The initial conditions in the circumstellar disk, the process of planet formation, and subsequent dynamical evolution undoubtly sculpt an individual distribution of small bodies for each planetary system. A recent analysis of the debris disk around $\tau$ Ceti (Greaves et al. 2004) suggests that the disk has similar dimensions, but mass an order of magnitude greater than the Kuiper Belt, indicating a more intense flux of cometary bodies. Is seems that, again, our solar system is not necessarily to be taken as the prevalent specimen, and that the bombardment by small bodies may become an important variable when searching for habitable Earth-like planets. Direct observations of debris disks are currently limited to very nearby stars, and are unavailable for the majority of extrasolar planetary systems found by radial velocity measurements (http://www.obspm.fr/planets). For these systems, models of planet formation could be used to produce a hypothetical distribution of small bodies, but this would lead us to undesirable complex simulations and heavy computational load. Hence, we accept the fact that their current population of small bodies is unknown, and suggest another kind of method of analysis.

In our approach we follow the evolution of a specific hypothetical population of small bodies which is common to all of the planetary systems under study. Our purpose is to isolate and characterize the small-body scattering properties of the planets, and to provide a technique to compare different planetary systems in this respect. If we can show that a certain configuration of planets is considerably more efficient in scattering small bodies than another one, we could argue that the actual small-body flux is less intense in that system, taken that the initial conditions in both systems are similar. Wetherill (1994) used this idea when he considered alternative giant-planet formation scenarios in our Solar System. UsingÖpik-Arnold calculations he followed the evolution of cometary test bodies and concluded that the absence of Jupiter would increase the cratering rate on Earth throughout its history by a factor of 100-1000. The dynamically dominant role of Jupiter in the Solar System was also shown byEverhart (1968), in the case of parabolic comets.

In this paper we will present computational tools to measure the gravitational scattering properties of a planetary system. First, in Sect. 2, we will introduce a "benchmark" integration scenario with thousands of cometary small bodies to be integrated numerically along the planetary system under study. The small bodies are initially in orbits that cross the HZ of the system. We will also define a variable, the escape rate, that describes 
the strength at which the planetary system scatters the small bodies into orbits that no longer threaten the HZ. In Sect. 3, we will present some preliminary results obtained using the tools above. We have considered hypothetical giant-planet configurations based on the Solar System and especially evaluated the dynamical significance of Jupiter. Later, we will show some results on selected extrasolar planetary systems. In Sect. 4 we will discuss the interpretation of the results.

\section{Methods}

\subsection{The escape rate of particles}

We study planetary systems of one or more giant planets orbiting a single Sun-like star. Into the planetary system we place a swarm of massless test particles representing a population of HZ-threatening small bodies. The initial orbital distribution of the particles is random, but chosen such that the pericenter distances are inside the HZ. As the system is propagated in time by numerical integration, the orbits of the particles are perturbed by the giant planets, deviating some of the particles into orbits that are no longer HZ-threatening. Our assumption is that this gravitational scattering is characteristic to the giant-planet system, and that its strength can be measured straightforwardly, as follows.

Suppose that in the scenario described above, we are in a state where the probability of a particle to be scattered per unit time, $\gamma$, is constant. This implies that at any time $t$ the size of the particle population $N$ changes by $\mathrm{d} N=-N \gamma \mathrm{d} t$, and that the population depletes exponentially;

$N(t)=N_{0} \mathrm{e}^{-\gamma\left(t-t_{0}\right)}$,

where $N_{0}$ is the initial population at time $t_{0}$. We could try to determine $\gamma$ simply by integrating the system from $t_{0}$ to $t$ and using (1), but some difficulties would arise: first, a suitable choice for $N_{0}$ and $t-t_{0}$ would depend on $\gamma$, i.e., on the scattering properties of the planetary system. Second, in reality, it is unlikely that we would see exactly exponential depletion of particles with small values of $N$. Because of these issues, we define $\rho$, an approximation to $\gamma$, similarly to the method of computing Liapunov characteristic exponents (LCEs) (see, e.g., Lichtenberg \& Lieberman 1992), as

$\rho=\frac{1}{\left(t-t_{0}\right)}\left(\sum_{i=1}^{k} \ln \frac{N_{0}}{N\left(t_{i}\right)}+\ln \frac{N_{0}}{N(t)}\right)$,

where $t_{0}<t_{1}<\ldots<t_{k}<t$. At the intermediate time points $t_{1}, \ldots, t_{k}$ the population is renormalized (regenerated) back to its initial level $N_{0}$ by creating new random particles from the initial orbital distribution. In this way we can keep $N(t)$ always inside predefined boundaries and select a common $N_{0}$ and $t-t_{0}$ for all the planetary systems we investigate. If a system scatters particles at a constant rate, we should see $\rho$ converging towards a specific value. We refer to this value as the escape rate. There is a resemblance to the escape-rate formalism in statistical mechanics (e.g., Dorfman 1999). We emphasize the analogy between the computation of $\rho$ and LCEs. Instead of exponential divergence of nearby orbits, we monitor the exponential depletion rate of particles in a large population. The renormalization is done for similar reasons in both cases; with $\rho$ its purpose is to exclude possible effects caused by the population not being "large" anymore.

We would also like to point out that our intention is to define the escape rate as a tool for statistical physics rather than as
Table 1. Initial orbital elements for the particle swarm.

\begin{tabular}{lcl}
\hline \hline Orbital element & & Interval \\
\hline pericenter distance & $q$ & {$[0.5,1.5] \mathrm{AU}$} \\
apocenter distance & $Q$ & {$[10,80] \mathrm{AU}$} \\
inclination & $\iota$ & {$[0, \pi]$} \\
longitude of the ascending node & $\Omega$ & {$[0,2 \pi]$} \\
longitude of perihelion & $\tilde{\omega}$ & {$[0,2 \pi]$} \\
mean longitude & $L$ & {$[0,2 \pi]$} \\
\hline
\end{tabular}

a measurement for actual physical quantities. Numerically computed escape rate values always depend on the initial orbital parameter distribution of the particle swarm. Therefore, the escape rate tells us how the planetary system responds to a certain type of initial population. In a specific application, in order to improve the informative value of the escape rate, the population could be chosen to represent, e.g., HZ-crossing comets originating from an Oort-type reservoir. In this paper, however, we choose an initial population which makes the demonstration of our method as simple as possible, but still allows us to make indicative arguments about real planetary systems.

\subsection{Parameters for the particle swarm}

In this section we define the initial and critical orbital elements for the particle swarm, and the dimensions of the HZ. These parameters fix a benchmark integration scenario which we use for computing escape rates in this paper.

Obtaining proper convergence in escape rate computation requires that the flux of particles throughout the planetary system remains as close to steady state as possible. If the initial orbital distribution that feeds new particles into the system is far away from the steady-state distribution, we see a secular drift in the escape rate value. In the process of finding a suitable and simple orbital distribution we learned that, in order to enhance the convergence of the escape rate computation, we should:

- choose an initial orbital distribution that is relatively homogeneous and contains only chaotic orbits. In other words, orbits that have strong interaction with the giant planets and a mutually similar timescale for being scattered;

- select an initial orbital distribution that is spherically symmetric. A disk-shaped distribution is not close to steady state, since many particles are perturbed into high inclination orbits before being scattered;

- introduce an offset time to the integration, after which the escape rate computation is started. This eliminates the effect of an initial transient period when the flux of particles seeks its equilibrium.

Following these guidelines, we define the initial particle population for our benchmark integration; each orbital element is randomly selected from an even distribution bounded by the intervals in Table 1 . The interval for pericenter distance $q$ coincides with our definition for the inner (0.5 AU) and outer (1.5 AU) edges of the HZ. This is a rough approximation; a detailed analysis of HZs around main sequence stars is given by Kasting et al. (1993). We adopt such a broad range of values since we want it to cover the actual HZs of most of the observed extrasolar systems. In addition, by using a common definition for the $\mathrm{HZ}$ we can compare the escape rates of systems with different central stars.

During the integration, particles scattered into orbits that will no longer cross the $\mathrm{HZ}$ should contribute to the escape rate value 
Table 2. Integration parameters in benchmark runs.

\begin{tabular}{lll}
\hline \hline number of particles & $N_{0}$ & 10000 \\
integration time & $t$ & 100000 years \\
escape rate offset time & $t_{0}$ & 50000 years \\
leapfrog step size & $\tau_{\text {lf }}$ & $\sim 40$ days \\
Bulirsch-Stoer step size & $\tau_{\mathrm{BS}}$ & $\sim 0.05-5$ days \\
\hline
\end{tabular}

and be removed. We define and monitor the following alternative criteria for particle removal:

- pericenter distance $q>2.0 \mathrm{AU}$

- ejection out of the system (semi-major axis $a>10000 \mathrm{AU}$ or hyperbolic orbit);

- collision with one of the planets or the central star.

\subsection{Orbit integration}

The escape rate computation requires a numerical integration method that has the following properties:

- the integrator must be able to handle close encounters between planets and massless bodies;

- high numerical accuracy is not important, but the method must be robust;

- the method has to be fast and optimized for integrating a planetary system with thousands of massless bodies.

We use a hybrid integration scheme where the primary propagator is the mixed variable symplectic (MVS) leapfrog method (see, e.g., Murray \& Dermott 1999). Close encounters are handled with the standard Bulirsch-Stoer algorithm (Stoer \& Bulirsch 1992) using the full force function. Our integration method is close to the method presented by Chambers (1999), the main difference being that we use heliocentric and Jacobian coordinates, and have not implemented a changeover function for more sophisticated switching between the integration methods. Since our close encounters are always between a massive and a massless body, the Hamiltonian is trivially conserved. Table 2 lists the integration parameters we use in the benchmark integration.

We have implemented the integrator in standard Fortran 95 using object-oriented programming techniques. It is part of a larger numerical software package that we intend to make public in the future. The Finnish Center of Scientific Computing (CSC) provides us computing time on their IBM eServer Cluster 1600, a supercomputer with 512 Power4-processors. A benchmark run on a single processor takes $6-8 \mathrm{~h}$ of CPU-time.

Since the massless particles do not interact with each other, the integration algorithm can be parallelized by distributing the particles evenly between the available processing units. In test runs, we have obtained a speedup factor of one magnitude by using 16 CPUs instead of one. However, with our current parameters, it is more efficient to run multiple benchmarks simultaneously, each with a single processor.

\section{Results}

\subsection{Integration consistency}

Before the scientific runs, we analysed the consistency of our integrator. We used two different test setups in order to determine the integration errors separately for planets and massless particles. In the first test, we integrated the four giants planets of the

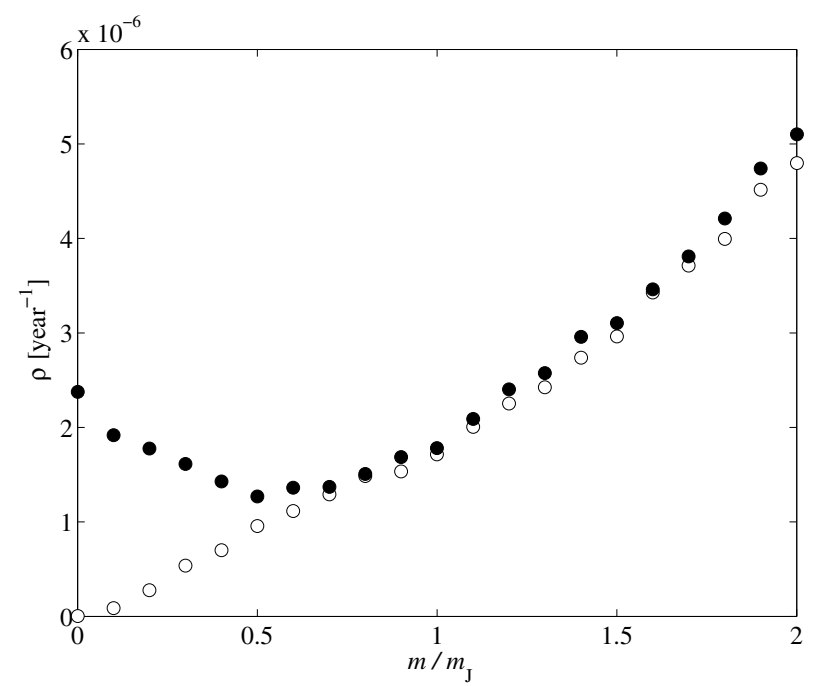

Fig. 1. Escape rates when Jupiter's mass is varied. System of Jupiter, Saturn, Uranus, and Neptune is shown with filled circles, and system of Jupiter alone with empty circles. $m_{\mathrm{J}}$ is the true mass of Jupiter.

Solar System for one million years (the step sizes were set according to Table 2). This was effectively a test for the MVS-part of the integrator, since there are no mutual close encounters between the planets. The relative energy error of our implementation shows similar behaviour to previously published ones (e.g., Chambers 1999), that is, it hovers around $10^{-7}$ throughout the integration time.

For the second test, we set up a system with the Sun, Jupiter, and 100 massless particles corresponding to the restricted threebody problem. Otherwise, the parameters were the same as in the benchmark runs (Table 2). We monitored the Jacobi constants of the particles and found that the average error was $7 \times 10^{-6}$ and the maximum $2 \times 10^{-4}$. By reducing all the step sizes by a factor of ten, the average and maximum errors changed to $5 \times 10^{-9}$ and $2 \times 10^{-7}$, respectively. For performance reasons, however, we decided that the step sizes in Table 2 are sufficient for our qualitative analysis.

\subsection{The role of Jupiter}

We have computed escape rate values in order to study the dynamical significance of Jupiter in the Solar System. We used two giant-planet configurations; one with all of the giants (Jupiter, Saturn, Uranus, and Neptune), and the other with Jupiter alone. The initial values for the planets were given at J2000 epoch. In both configurations, we varied the mass of Jupiter while retaining all other parameters. The computed escape rate values are plotted in Fig. 1.

In the single planet case, we see close to linear dependence between the mass of Jupiter and the escape rate. It is not surprising to notice that a more massive planet scatters particles more efficiently, but the responsiveness of the escape rate value encourages us to believe that it could indeed possess some informative value. The convergence of each escape rate computation in the single planet case can be can be seen from Fig. 2. It seems that our choices for the integration time and the number of particles are sufficient, at least in this case.

Returning to Fig. 1, to the case where all four giant planets are present, we now see more details in the escape rate. When the mass of Jupiter is larger than its true value $1.0 \mathrm{~m}_{\mathrm{J}}$, the escape rate behaves similarly to the system with Jupiter alone. 


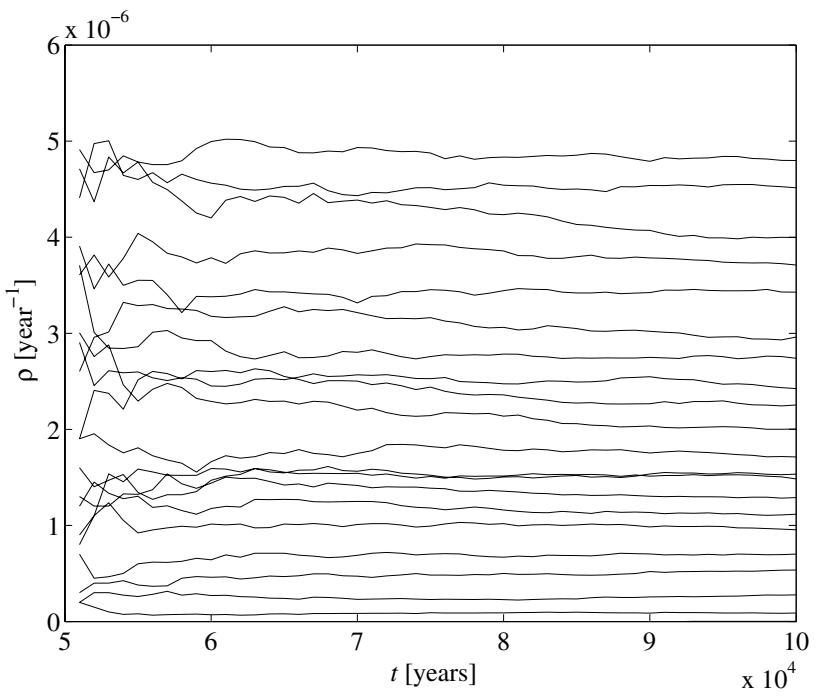

Fig. 2. Convergence of the escape rate values in integrations with Jupiter alone (cf. Fig. 1).

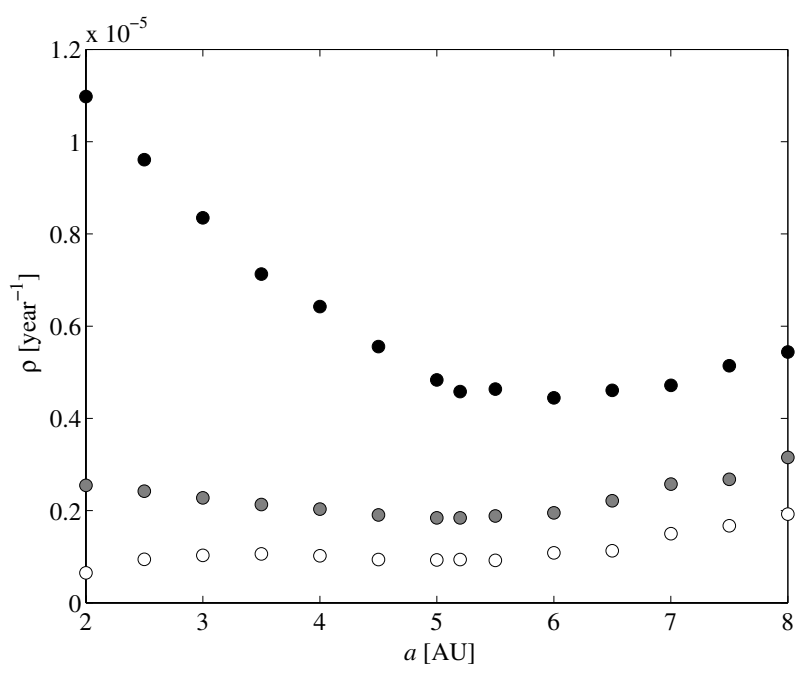

Fig. 3. Escape rates for the Jupiter-only case when the semi-major axis $a$ of the planet is varied. The mass of Jupiter is $2.0 \mathrm{~m}_{\mathrm{J}}$ (black circles), $1.0 m_{\mathrm{J}}$ (gray circles), and $0.5 m_{\mathrm{J}}$ (empty circles).

This can be interpreted as Jupiter being the dominant scatterer of particles among the other giants. However, for Jupiter masses below $1.0 \mathrm{~m}_{\mathrm{J}}$, the four-giant case eventually separates from the Jupiter-only case, and the other giants take over in particle scattering, effectively replacing Jupiter as its mass goes to zero. There is a minimum in the escape rate value which roughly coincides with the point of separation. We do not have a proper explanation to this phenomenon, but one should consider the following detail: the masses of Saturn $\left(m_{\mathrm{S}}\right)$, Uranus $\left(m_{\mathrm{U}}\right)$, and Neptune $\left(m_{\mathrm{N}}\right)$ satisfy $m_{\mathrm{S}}+m_{\mathrm{U}}+m_{\mathrm{N}} \approx 0.4 m_{\mathrm{J}}$. Hence, when the mass of Jupiter is $0.6 m_{\mathrm{J}}$ the total mass of the four giants is approximately $1.0 \mathrm{~m}_{\mathrm{J}}$. We cannot confirm that this value is somehow special for the system, but interestingly, it approximately coincides with the point of separation and the minimum.

Besides the mass, we were interested how changes in the semi-major axis of Jupiter would affect the escape rate. We took the system with Jupiter alone with three different masses $\left(0.5 \mathrm{~m}_{\mathrm{J}}\right.$, $1.0 m_{\mathrm{J}}, 2.0 \mathrm{~m}_{\mathrm{J}}$ ) and varied the semi-major axis of the planet (Fig. 3).

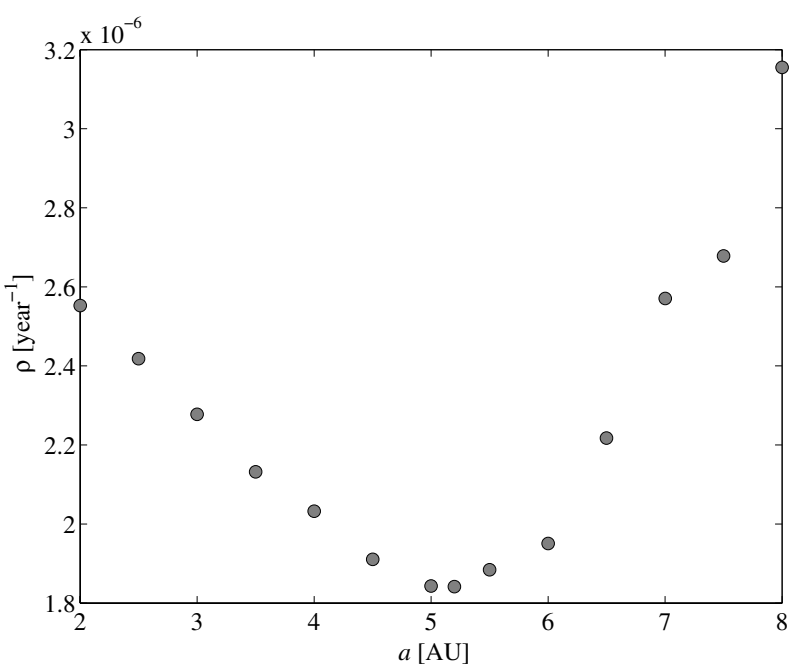

Fig. 4. Escape rates for the Jupiter-only case $\left(1.0 \mathrm{~m}_{\mathrm{J}}\right.$, isolated from Fig. 3) when the semi-major axis $a$ of the planet is varied.

Table 3. Initial parameters for the extrasolar planets.

\begin{tabular}{lllll}
\hline \hline Parameter & 47 UMa b & 47 UMa c & GJ 777A c & GJ 777A b \\
\hline$a[\mathrm{AU}]$ & 2.09 & 3.73 & 0.128 & 3.92 \\
$e$ & 0.061 & 0.1 & 0.01 & 0.36 \\
$\tilde{\omega}\left[^{\circ}\right]$ & 172.0 & 127.0 & 153.7 & 12.4 \\
\hline$m\left[m_{\mathrm{J}}\right]$ & 2.54 & 0.76 & 0.057 & 1.502 \\
\hline
\end{tabular}

It seems that increasing the mass of the planet has greater impact on the scattering strength than varying its semi-major axis. At least with larger masses of Jupiter $\left(1.0 \mathrm{~m}_{\mathrm{J}}\right.$ and $\left.2.0 \mathrm{~m}_{\mathrm{J}}\right)$, we can identify a minimum in the escape rate occurring at a certain value of the semi-major axis. The case where Jupiter has its true mass is isolated in Fig. 4. It is interesting to notice that the minimum occurs approximately at 5.2 AU, at the true semi-major axis of Jupiter.

By monitoring the orbital parameters of the scattered particles we can identify two trends that, when combined, explain the minimum. Remembering our criteria for removing particles from integration, we see that a Jupiter with $a>5.2 \mathrm{AU}$ is increasingly effective at pulling the pericenter distances of particles above the 2.0 AU limit. On the other hand, the closer a giant planet is to the $\mathrm{HZ}$ the stronger are the perturbations on particles near their pericenters, resulting in numerous ejection orbits.

\subsection{Extrasolar systems}

We computed the escape rate values for two known extrasolar planetary systems; 47 UMa and GJ 777A (HD 190360). Table 3 shows our initial parameters for the planets. The data for $47 \mathrm{UMa}$ is given by Fischer et al. (2002). The system is one of the best candidates for having earth-like planets. Until recently, GJ 777A was also considered as a feasible candidate. However, measurements by Vogt et al. (2005) may change this picture, since they suggest that there is a second planet orbiting close to the central star. Nevertheless, we chose to analyse GJ 777A, because it now represents a qualitatively different case where the HZ lies between the orbits of two perturbing planets.

Inclinations $\iota$, longitudes of the ascending node $\Omega$, and mean longitudes $L$ were all set to zero. Masses of the central stars were $1.03 m_{\odot}$ for $47 \mathrm{UMa}$ and $0.96 m_{\odot}$ for GJ 777A. The 
Table 4. Escape rates for selected extrasolar systems.

\begin{tabular}{lc}
\hline \hline Planetary system & $\rho\left[\right.$ year $\left.^{-1}\right]$ \\
\hline Solar System & $1.78 \times 10^{-6}$ \\
47 UMa & $2.08 \times 10^{-5}$ \\
GJ 777A & $4.25 \times 10^{-5}$ \\
\hline
\end{tabular}

computed escape rate values are shown in Table 4, and compared to the Solar System (with four giants).

\section{Discussion}

We have introduced a technique for measuring gravitational scattering efficiency in a planetary system. One simple dynamical quantity, the escape rate, describes the protection that a giantplanet system offers to a hypothetical terrestrial planet. Our results show that, at least with our current choice of parameters, the escape rate is a computationally consistent quantity and is a function of the orbital structure of the giant planets. The important question is: can we use escape rates to make conclusions that apply to real planetary systems or are they just a dynamical curiosity with no physical significance?

We believe that the very definition for the escape rate (Sect. 2.1) is rigorous and, when comparing planetary systems, the one with a higher escape rate would be more efficient in scattering small bodies away from the HZ. However, this argument applies only to the particular initial population of small bodies used in the escape rate scenario. Hence, it is the choice of the population that mostly determines what the escape rate really tells us.

There is an obvious conceptual difference that should be noted. In reality, the shape and intensity of the small-body flux in a planetary system is a function of time, whereas in the escape rate computation, the flux is intentionally kept constant. Therefore, each escape rate scenario represents a fixed moment in time. In order to simulate the small-body flux in a real planetary system, we should fix a time in the history (or in the future) of the system, and create an initial population that is a model for the HZ-crossing flux at that particular moment. Unfortunately, by doing so, we would inevitably lose the ability to compare the escape rates of totally different planetary systems. This kind of approach could still be valid and beneficial, if we concentrated to a one well known system (i.e., to our Solar System) and varied the parameters of the planets only by small amounts.

In the benchmark integration scenario presented in this paper (Sect. 2.2) we use the escape rate as a universal dynamical quantity which is not identified with real fluxes of small bodies. Our initial population is artificial, but for the sake of creditability, it should still be somehow "typical" among planetary systems. Our distribution of particles is spherically symmetric, and in the orbital parameter space the particles cover the angular dimensions $(\iota, \Omega, \tilde{\omega}, L)$ completely, but only a limited range in semi-major axis and eccentricity $(a, e)$. This kind of distribution is not typical to the observed small-body populations or to the simulations explaining their evolution (see, e.g., Duncan et al. 1987, 1988, for Solar System comets). However, one should remember that the initial population for the escape rate is supposed to represent only the fraction of small bodies that threaten the HZ of the planetary system. The modelling of all the processes that bring small bodies into to HZ-crossing flux would be a cumbersome task and, hence, we feel justified to use our simplified initial population as a first order approximation.
As a part in improving the convergence and efficiency in escape rate computations, we chose the initial orbital distribution in a way that the apocenter distances $(Q)$ were all well beyond the semi-major axis of the innermost giant planet. Obviously, a qualitatively different choice, e.g., one with the apocenter distances inside the orbits of the giants, could change our results (Sect. 3) significantly. This is something we wish to investigate in future papers. However, as an indication of partial robustness, the qualitative behaviour of our results did not change when we used an initial population with $Q=[40,50]$ and a narrower definition for the $\mathrm{HZ}$ and for the removal criterion by particle pericenter $([0.8,1.4]$ and $1.5 \mathrm{AU}$, respectively). Our choice for the initial population also ensures that the orbits of the particles are chaotic and none of the particles survive the whole integration time. If the population included numerous stable or resonant orbits, the regenerated particles might accumulate into safe areas in the phase space, and the escape rate might become biased.

There are open questions about the initial population, but our results with the benchmark scenario show that the escape rate has informational value, and it can be used in comparative analyses on planetary systems which was our goal in the first place. As such, the escape rate cannot predict the habitability of a hypothetical terrestrial planet in an observed extrasolar planetary system, but it could be used, among other tools, to make educated guesses about the matter.

The computed escape rate values for the Solar System giant planets confirm that Jupiter has the dominant role in scattering small bodies out of the HZ. The scattering efficiency in the Solar System would not be greatly disturbed if the other giant planets were removed. This is in line with the findings by Wetherill (1994). On the other hand, removing Jupiter does not affect the escape rate considerably either since the other giants effectively take its role in scattering.

The minimum in the escape rate at the true semi-major axis of Jupiter is an interesting detail. It could be a coincidence, but perhaps there is some cosmogonical, or dynamical, explanation. Maybe Jupiter, the dominant mass, is in an optimal orbit to encourage the heavy bombardment of cometary bodies into the inner Solar System. This could have been an essential requirement for life and evolution on Earth.

If we compare the escape rate for the Solar System to the escape rates for $47 \mathrm{UMa}$ and GJ 777A we see that small-body scattering efficiency is more than an order of magnitude greater in the extrasolar systems. A natural explanation to this are the smaller semi-major axes and greater masses of the extrasolar giant planets. Comparison between $47 \mathrm{UMa}$ and GJ 777A is more interesting, because the higher escape rate of GJ 777A is difficult to explain by intuition. By looking at the statistics produced by the integration, we saw that the outer planet in GJ 777A was the most recent perturber for $92 \%$ of the scattered particles. Therefore, the large eccentricity of the outer planet is probably responsible for the higher escape rate value.

This is a preliminary paper where we have introduced a new concept, implemented a computational method, and demonstrated some basic applications. There are many possibilities for follow-up studies; an important one is to analyse further the effect of the initial population on the escape rate. Another task, which was beyond the CPU-time budget of this paper, would be to probe the parameter space of the Solar System giants more thoroughly. In addition to semi-major axes and planetary masses, one could include the eccentricities into the analysis. Generally, the increasing dimensionality of the parameter space will certainly become a problem, but with some kind of compromises, 
one could compute escape-rate maps which could reveal interesting dynamical structures.

Acknowledgements. This work was partly supported by the Finnish Cultural Foundation, Emil Aaltonen Foundation, Jenny and Antti Wihuri foundation, and Academy of Finland. We are grateful to an anonymous referee who provided us with thorough and constructive comments.

\section{References}

Asghari, N., Broeg, C., Carone, L., et al. 2004, A\&A, 426, 353

Barnes, R., \& Quinn, T. 2004, ApJ, 611, 494

Chambers, J. E. 1999, MNRAS, 304, 793

Dorfman, J. R. 1999, An Introduction to Chaos in Nonequilibrium Statistical Mechanics (Cambridge University Press)
Duncan, M., Quinn, T., \& Tremaine, S. 1987, 94, 1330

Duncan, M., Quinn, T., \& Tremaine, S. 1988, ApJ, 328, L69

Everhart, E. 1968, 73, 1039

Fischer, D. A., Marcy, G. W., Butler, R. P., Laughlin, G., \& Vogt, S. S. 2002, ApJ, 564, 1028

Greaves, J. S., Wyatt, W. S., Holland, W. S., \& Dent, W. R. F. 2004, MNRAS, 351, L54

Jones, B. W., Underwood, D. R., \& Sleep, P. N. 2005, ApJ, 622, 1091

Kasting, J. F., Whitmire, D. P., \& Reynolds, R. T. 1993, 101, 108

Lichtenberg, A. J., \& Lieberman, M. A. 1992, Regular and Stochastic Dynamics (Springer)

Menou, K., \& Tabachnik, S. 2003, ApJ, 583, 473

Murray, C. D., \& Dermott, S. F. 1999, Solar System Dynamics (Cambridge University Press)

Stoer, J., \& Bulirsch, R. 1992, Introduction to Numerical Analysis (Springer)

Vogt, S. S., Butler, R. P., Marcy, G. W., et al. 2005, ApJ, 632, 638

Wetherill, G. W. 1994, Ap\&SS, 212, 23 